\title{
28 Research Square \\ Is Primary Chemoradiation A Better Treatment? A Retrospective Study of Early Stage Node-positive Cervical Cancer.
}

\author{
Nan Zhang \\ Beijing Cancer Hospital \\ Hong Zheng ( $\square$ zheng_111_hong@126.com ) \\ Beijing Cancer Hospital
}

\section{Research article}

Keywords: cervical cancer, lymphatic metastasis, radical hysterectomy

Posted Date: February 7th, 2020

DOI: https://doi.org/10.21203/rs.2.22834/v1

License: (c) (i) This work is licensed under a Creative Commons Attribution 4.0 International License.

Read Full License

Version of Record: A version of this preprint was published at Clinical and Experimental Obstetrics \& Gynecology on January 1st, 2021. See the published version at https://doi.org/10.31083/j.ceog4806216. 


\section{Abstract}

Background. Cervical cancer is the second most frequently diagnosed cancer and the third leading cause of cancer death for women in developing countries. Radical hysterectomy with bilateral pelvic lymph node dissection is usually preferred for patients of stage IB1-IIA2. Currently, image examinations have certain limitations in diagnose of lymph node metastasis and their detection accuracies are not satisfactory. Only the pathological examination after removal of the suspected metastatic lymph nodes during surgery can conclusively identify the presence of metastasis. If there is a positive result of lymphatic metastasis, there is no clear guideline whether to complete a radical surgery, or to only conduct a systematic lymphadenectomy, followed with adjuvant Concurrent Chemoradiotherapy (CCRT). This retrospective study aimed to compare the efficacy and safety of the two treatment modalities. Methods. 49 stage IB1-IIA2 cervical cancer patients with lymphatic metastasis confirmed by systemic pelvic and para-aortic lymph node dissection from 2007 to 2018 were reviewed. The patients were treated with either primary chemoradiation or radical hysterectomy followed by adjuvant chemoradiation after lymphadenectomy. Survival states and adverse events of the two treatments were compared. Results. Median follow-up time was 45 (range 11-119 months) months. In non-radical surgery group, 1 patient $(1 / 15,6.7 \%)$ relapsed and died, while in radical surgery group, 7 patients $(7 / 27,25.9 \%)$ relapsed and 5 $(5 / 27,18.5 \%)$ died. Significant difference was found in the mean progression-free survival between the two groups, which was $69(95 \% \mathrm{Cl} 49.118-88.882)$ months in non-radical surgery group and $44(95 \% \mathrm{Cl}$ 35.857-52.143) months in radical surgery group ( $\mathrm{p} \otimes 0.01$ ). There was significant difference in three-year progression-free survival( $86 \% \mathrm{vs} .71 \%, \mathrm{p} \otimes 0.01)$. Grade 3-4 toxicity was comparable between the two groups ( $26.7 \%$ vs. $25.9 \%, p=0.958)$. Conclusion. For stage IB1-IIA2 cervical cancer patients with positive lymph node, primary chemoradiation after pelvic and para-aortic lymphadenectomy seems to have better survival outcomes compared with radical hysterectomy by laparoscopy plus chemoradiation in the retrospective study with limited cases. Evidence from a randomized controlled study is in need to confirm the optimal treatment for early stage node-positive cervical cancer.

\section{Background}

Cervical cancer is the fourth most frequently diagnosed cancer and the fourth leading cause of cancer death in women, with estimated 570000 diagnosed cases and 311000 deaths in 2018 globally ${ }^{[1]}$. In China, it is the sixth most common malignancy, the eighth most common cause of cancer death in women and the first most common cause of gynecologic cancer death ${ }^{[2]}$. Lymphatic metastasis is one of the most important prognostic factors in cervical cancer patients ${ }^{[3]}$. Surgical treatment is usually preferred for patients with cervical cancer in stage IB1-IIA2, especially in women of childbearing age ${ }^{[4]}$. Patients with surgically confirmed lymphatic metastasis are suggested to receive concurrent chemoradiotherapy (CCRT) with platinum ${ }^{[4]}$. Nowadays, radiologic examinations such as Computed Tomography (CT), Magnetic Resonance Imaging (MRI) and Positron Emission Tomography (PET) are allowed to be performed before treatment if available. However, the accuracy of the evaluation of lymph node metastasis is not satisfactory. It was reported that the sensitivity to detect lymph node metastasis 
was $57 \%$ for CT, $75 \%$ for MRI, and $68 \%$ for PET, while the specificity was $91 \%$ for CT, $75 \%$ for MRI, and $72-84 \%$ for PET ${ }^{[5]-[7]}$. Only the pathological examination after removal of the suspected metastatic lymph nodes during surgery can clearly identify the presence of metastasis. If there is a positive result of lymphatic metastasis, there is no conclusive proof whether to continue to complete the radical surgery, or to just complete the systematic lymphadenectomy, followed with adjuvant CCRT. This retrospective study aimed to compare the efficacy and safety of the two treatment modalities.

\section{Methods}

49 cases of stage IB1-IIA2 cervical cancer patients who received surgical treatment in Peking University Cancer Hospital during October 2007 to September 2018 were reviewed. The preoperative staging was according to the Federation International of Gynecology and Obstetrics(FIGO) 2009 standards. All patients enrolled underwent a systematic pelvic lymph node dissection and were confirmed of lymph node metastasis by rapid frozen pathological examination during the surgery. The patients were divided into two groups according to their different treatments: radical surgery (RS) group and non-radical surgery (NRS) group.

For patients in RS group, radical hysterectomy after systemic pelvic lymph nodes excision by laparoscopy were performed, while in NRS group, their radical surgeries were abandoned after systemic pelvic lymph nodes excision and para-aortic lymph node dissection through laparoscopy or extraperitoneal approach. Patients in both groups received adjuvant CCRT after the surgery.

Synchronous chemotherapy regimen was cisplatin, $40 \mathrm{mg} / \mathrm{m}^{2}$, with a maximum dose of $60 \mathrm{mg}$ per week for a total of 6 weeks. The pelvic radiotherapy dose was 45-50Gy, with comprehensive coverage of tumor beds and pelvic lymph nodes. And the intracavity radiotherapy dose was 10-12Gy at A point in RS group and 30-35Gy at A point in NRS group.

Follow-up with the patients was conducted since the surgery by looking up in their clinical records or phone calls. The latest follow-up was in July 2019.

The endpoint was progression-free survival (PFS), which was defined as the first observation of local recurrence or distant metastasis. PFS was estimated by Kaplan-Meier method, and the difference between the two groups was compared by log-rank test in univariate analysis. The comparison of the counting data was tested by $\chi 2$ test. Statistical analysis was performed using SPSS 21.0 (SPSS Inc., Chicago) with a significance level of $p=0.05$.

\section{Results}

7 of the 49 patients were lost to follow-up, and the clinical pathological characteristics of the remaining 42 patients are summarized in Table 1. No significant difference in the clinical pathological characteristics between the two groups was found. The median follow-up time was 45 months (range from 11 to 110 months). Follow-up time of the NRS and RS groups were 69 and 38 months, respectively, 
with a significant difference between the two groups. Recurrences were observed in 8(8/42) patients, from which 1 case of pelvic recurrence and distant metastasis in NRS group, and all the other 7 cases in RS group. 1 patient in NRS group and 5 patients in RS group died of relapse. Recurrence and metastasis in both groups are shown in Table 2 .

Table 1

Patient characteristics.

\begin{tabular}{|c|c|c|c|c|}
\hline Characteristics & $\operatorname{ALL}(n=42)$ & $\operatorname{NRS}(n=15)$ & $R S(n=27)$ & $\mathbf{P}$ \\
\hline $\begin{array}{l}\text { Age,years } \\
\text { Median (std) }\end{array}$ & $41.4(6.28)$ & $42.5(4.76)$ & $40.6(7.13)$ & 0.967 \\
\hline Histology & & & & 0.655 \\
\hline SCC & $34(81.0 \%)$ & $13(86.6 \%)$ & $21(77.8)$ & \\
\hline$A C$ & $5(11.8 \%)$ & $1(6.7 \%)$ & $4(14.8 \%)$ & \\
\hline ASC & $2(4.8 \%)$ & $1(6.7 \%)$ & $1(3.7 \%)$ & \\
\hline OTHER & $1(2.4 \%)$ & $0(0 \%)$ & $1(3.7 \%)$ & \\
\hline Pathological grade & & & & 0.817 \\
\hline I & $1(2.4 \%)$ & $1(6.7 \%)$ & $0(0 \%)$ & \\
\hline II & $19(45.2 \%)$ & $3(20 \%)$ & $16(59.3 \%)$ & \\
\hline III & $22(52.4 \%)$ & $11(73.3 \%)$ & $11(40.7 \%)$ & \\
\hline Tumor size(cm) & & & & 0.439 \\
\hline$\varangle 4$ & $7(16.7 \%)$ & $2(13.3 \%)$ & $5(18.5 \%)$ & \\
\hline$\leq 4$ & $35(83.3 \%)$ & $13(86.7 \%)$ & $22(81.5 \%)$ & \\
\hline FIGO stage & & & & 0.381 \\
\hline IB1 & $27(64.3 \%)$ & $10(66.7 \%)$ & $17(63.0 \%)$ & \\
\hline IB2 & $8(19.0 \%)$ & $3(20 \%)$ & $5(18.5 \%)$ & \\
\hline$\| \mathrm{A} 1$ & $5(11.9 \%)$ & $2(13.3 \%)$ & $3(11.1 \%)$ & \\
\hline IIA2 & $2(4.8 \%)$ & $0(0 \%)$ & $2(7.4 \%)$ & \\
\hline $\begin{array}{l}\text { Number of lymph node metastasis } \\
1 \\
\geq 2\end{array}$ & $\begin{array}{l}13(30.9 \%) \\
29(69.1 \%)\end{array}$ & $\begin{array}{l}5(33.3 \%) \\
10(66.7 \%)\end{array}$ & $\begin{array}{l}8(29.6 \%) \\
19(70.4 \%)\end{array}$ & 0.439 \\
\hline
\end{tabular}


Table 2

Patterns of recurrence and disease status

\begin{tabular}{|llll|}
\hline & No.of patients $(\mathbf{n}=\mathbf{4 2})$ & NRS( $\mathbf{n}=15)$ & RS $(\mathbf{n}=\mathbf{2 7})$ \\
\hline Recurrence & $8(19.0 \%)$ & $1(6.7 \%)$ & $7(25.9 \%)$ \\
\hline Local & - & - & - \\
\hline Distant & $5(11.9 \%)$ & - & $5(18.5 \%)$ \\
\hline Both & $3(7.1 \%)$ & $1(6.7 \%)$ & $2(7.4 \%)$ \\
\hline
\end{tabular}

The median PFS in NRS group and RS group were 69 months (95\% confidence interval (Cl) 49.12-88.88), and 44 months $(95 \% \mathrm{Cl} 35.86-52.14)$, with a significant difference $(P=0.019)$. The survival curve is shown in Fig. 1.

Significant difference of the three-year PFS rate between the two groups can be observed, with $86 \%$ in NRS group and $71 \%$ in RS group (pख0.01).

No death related to adverse events(AE) of the treatments was found. There was no significant difference in rate of Grade 3-4 toxicity ( $p=0.958)$. In NRS group, 2 cases of Grade $3-4$ hematological AE, 1 case of Grade 3 gastrointestinal AE and 1 case of vesicovaginal fistula were observed. While 4 cases of Grade 34 hematological $A E, 1$ case of Grade 3 gastrointestinal $A E, 1$ case of vesicovaginal fistula and 1 case of ureteral fistula showed up in RS group.

Table 3

Treatment-related toxicity

\begin{tabular}{|lllll|}
\hline CTCAE Grade $\geq 3$ toxicity & ALL & NRS & RS & P \\
\hline Blood & & & & $\mathbf{0 . 9 5 8}$ \\
\hline Gastrointestinal & $6(14.3 \%)$ & $2(13.3 \%)$ & $4(14.9 \%)$ & \\
\hline Genitourinary & $2(4.8 \%)$ & $1(6.7 \%)$ & $1(3.7 \%)$ & \\
\hline
\end{tabular}

\section{Discussion}

Lymph node metastasis is a crucial high-risk prognostic factor in early stage cervical cancer, as the fiveyear survival rate can decrease from $82-90 \%$ to $38-61 \%$ with the lymphatic metastasis ${ }^{[8]}$. The incidence of lymph node metastasis in stage IB and IIA cervical cancer are $12-22 \%$ and $10-27 \%$, respectively [9]-[12]. As positive lymph node plays an determining role in the adjuvant therapy, the FIGO 2018 staging standard brings lymph node metastasis as one of the staging factors. According to the standard, patients 
only with pelvic lymph nodes are staged as IIIC1, and are staged as IIIC2 if para-aorta lymph nodes involved ${ }^{[13]}$.

Currently, image examinations have certain limitations in diagnose of lymph node metastasis. A systemic lymph nodes dissection for pathological examination is necessary to acquire an accurate diagnose. Pelvic lymphadenectomy consisted of bilateral following regions: along the common iliac vessels, the external and internal iliac vessels until the crossing of the circumflex vein caudally, obturator fossa including the ventral and dorsal of the obturator nerve. In the study, para-aortic lymph node dissection was performed in NRS group and no metastasis was found.

According to the National Comprehensive Cancer Network (NCCN) guidelines, cervical cancer patients with lymph node metastasis should be treated with CCRT after surgery ${ }^{[4]}$. Local early cervical cancer patients may consider CCRT as an initial treatment regardless their condition of lymphatic metastasis, since its therapeutic effect is equivalent to surgery. And there is a controversy over whether to perform RS or not for local early cervical cancer patients with intra-operative lymph node metastasis. There are several retrospective studies evaluated the two treatment modalities. A retrospective multicenter cohort study analyzed 121 patients, 89 cases from which finished the RS with pelvic lymphadenectomy(RS Group), while the rest 32 cases received chemoradiotherapy after pelvic lymphadenectomy(NRS Group) ${ }^{[14]}$.There was no difference in OS between the two groups while five-year PFS was in favor of RS group $(81 \%$ vs $67 \%$ ). Grade $3-4$ toxicity rates were higher in NRS group ( $59 \%$ vs $30 \%$ ), mainly because of the difference in chemotherapy-related hematologic toxicity. Another retrospective study consisted of 163 cases in RS group and 55 cases in NRS group ${ }^{[15]}$, and no significant difference existed in OS or PFS. However, prognosis of NRS group seems to be better than RS group in this study with a longer PFS. The difference might due to the surgical approach. Owing to the absence of the surgical approach in the multicenter cohort study, the RS surgery could be performed by laparotomy or laparoscopic approach. In this study, all of the 27 patients in RS group and 11 patients in NRS group underwent laparoscopic surgery, and the other 4 cases in NRS group received extraperitoneal operation. Both the NCCN and ESGO (European Society of Gynecological Oncology) guidelines once recommended laparoscopic approach as an option for radical hysterectomy, on account of the minimal trauma and rapid recovery. However, at the 2018 International Gynecologic Cancer Society (IGCS) meeting, the LACC study led by M.D. Anderson Cancer Center in the United States, reported that for early cervical cancer patients, patients who underwent minimally invasive surgery had lower Disease-free survival (DFS) and OS, and higher recurrence rate in comparison to those underwent open abdominal operation ${ }^{[16]}$. Nevertheless, previous studies have shown that for early cervical cancer, laparoscopic lymph nodes dissection do not affect the prognosis compared to open abdominal surgery ${ }^{[17]}$. In this study, all patients in RS group underwent a laparoscopic surgery, which might lead to poor outcomes. Thus, open abdominal operation cases in RS group will be included in the future study.

Meanwhile, most of the recurrences in this study occurred within 2 years after surgery, suggesting that more intensified surveillance is in need in order to capture the early recurrence signs. 
Differences in the total dose of intracavity exposure may also contribute to the differences of the PFS between the two groups. In order to protect the intestine, bladder and other adjacent pelvic organs, the total dose of intracavity radiation in RS group was 10-12Gy, which was lower than that in NRS group, the patients of which have the retention of the cervix lesion with a total dose of intracavity exposure reached 30-35Gy.

Furthermore, patients in NRS group began to receive CCRT earlier after surgery (an average interval period of 27.6 days) than RS group (an average interval period of 42.5 days), due to their faster recovery. The patients in NRS group received CCRT more timely and may lead to improved prognosis.

As for adverse events, 2 patients in NRS group and 4 patients in RS group reported chemoradiotherapyrelated grade 3-4 hematologic toxicity. Grade 3 gastrointestinal reaction occurred one for each group, possibly associated with chemoradiotherapy. One case of vesicovaginal fistula showed in each group, with one month after the therapy in NRS group and at the end of the CCRT in RS group, believed led to radiotherapy. As for the operative complication, one patient in RS group had a ureter fistula right after the surgery. Regarding to the small number of cases in the two groups, further studies are in need to fully evaluate the adverse events.

In addition to the above-mentioned limitations, the follow-up time of this study is not long enough to evaluate the long-term survival rate, especially for RS group, and it has to be further followed up.

\section{Conclusions}

For local early cervical cancer patients with lymph node metastasis detected during surgery, CCRT without radical hysterectomy seems to be as safe as CCRT after radical surgery by laparoscopy, and to have an improved prognosis and survival results. However, no definite conclusion should be drawn before a well-designed prospective study in a large sample size.

\section{Declarations}

\section{Ethics approval and consent to participate}

Because this is only a systematic review of previous patients' information and does not involve any human experiment or animal experiment, ethics approval and consent are not applicable. The informed consent for the use of patient information was obtained.

\section{Consent for publication}

Not applicable.

\section{Availability of data and materials}


The datasets used and analyzed during the current study are available from the corresponding author on reasonable request.

\section{Competing interests}

The authors declare that they have no conflicts of interest.

\section{Funding}

Not applicable.

\section{Authors' contribution}

ZN: search, study selection, data collection, interpretation of results, manuscript writing.

ZH: search, study selection, manuscript writing.

Both authors have read and approved the manuscript.

\section{Acknowledgements}

Not applicable.

\section{Abbreviations}

CCRT

Concurrent Chemoradiotherapy

CT

Computed Tomography

MRI

Magnetic Resonance Imaging

PET

Positron Emission Tomography

FIGO

Federation International of Gynecology and Obstetrics

RS

radical surgery

NRS

non-radical surgery

PFS

progression-free survival

OS

overall survival

$\mathrm{Cl}$ 
confidence interval

$\mathrm{AE}$

adverse events

NCCN

National Comprehensive Cancer Network

ESGO

European Society of Gynecological Oncology

IGCS

International Gynecologic Cancer Society

DFS

Disease-free survival

\section{References}

[1]. Bray Freddie,Ferlay Jacques,Soerjomataram Isabelle et al. Global cancer statistics 2018: GLOBOCAN estimates of incidence and mortality worldwide for 36 cancers in 185 countries.[J] .CA Cancer J Clin, 2018, 68: 394-424.

[2]. Sun Ke-xin, Zheng Rong-shou, Zhang Si-wei et al. Report of cancer incidence and mortality in different areas of China,2015.[J]. China Cancer, 2019, 28(1):1-11.

[3]. Masayoshi Hosaka, Hidemichi Watari , Takashi Mitamura. Survival and prognosticators of nodepositive cervical cancer patients treated with radical hysterectomy and systematic lymphadenectomy. [J]. International Journal of Clinical Oncology 2011.16(1),33-38.

[4]. National Comprehensive Cancer Network. NCCN Clinical Practice Guidelines in Oncology: Cervical Cancer, V.5.2019.

[5]. Liu Bin, Gao Sujuan, Li Shuofeng, A Comprehensive Comparison of CT, MRI, Positron Emission Tomography or Positron Emission Tomography/CT, and Diffusion Weighted Imaging-MRI for Detecting the Lymph Nodes Metastases in Patients with Cervical Cancer: A Meta-Analysis Based on 67 Studies.[J] .Gynecol. Obstet. Invest., 2017, 82: 209-222.

[6]. Kan Yangyang, Dong Di, Zhang Yuchen et al. Radiomic signature as a predictive factor for lymph node metastasis in early-stage cervical cancer.[J] .J Magn Reson Imaging, 2019, 49: 304-310.

[7]. Papadia Andrea, Gasparri Maria Luisa, Genoud Sophie et al. The combination of preoperative PET/CT and sentinel lymph node biopsy in the surgical management of early-stage cervical cancer.[J] .J. Cancer Res. Clin. Oncol., 2017, 143: 2275-2281.

[8]. Delgado G:Stage IB squamous cancer of the cervix:The choice of treatment.[J]. Obstet Gynecol Surv.1978, 33:174-183. 
[9]. Kamura T, Tsukamoto N, Tsuruchi N, et al. Multivariate analysis of the histopathologic prognostic factors of cervical cancer in patients undergoing radical hysterectomy.[J]. Cancer 1992,69:181-186.

[10]. Lai $\mathrm{CH}$, Chang HC, Chang TC, et al. Prognostic factors and impacts of adjuvant therapy in earlystage cervical carcinoma with pelvic node metastases.[J]. Gynecol Oncol 1993,51:390-396.

[11]. Benedetti-Panici P, Maneschi F, Scambia G, et al. Lymphatic spread of cervical cancer: an anatomical and pathological study based on 225 radical hysterectomies with systematic pelvic and aortic lymphadenectomy. [J].Gynecol Oncol 1996,62:19-24.

[12]. Sakuragi N, Satoh C, Takeda N, et al. Incidence and distribution pattern of pelvic and paraaortic lymph node metastasis in stage IB, IIA and IIB cervical carcinoma treated with radical hysterectomy.[J] Cancer 1999,85:1547-1554.

[13]. Bhatla Neerja, Aoki Daisuke, Sharma Daya Nand et al. Cancer of the cervix uteri.[J] .Int J Gynaecol Obstet, 2018, 143: 22-36.

[14]. Derks Marloes, Groenman Freek A, van Lonkhuijzen Luc R C W et al. Completing or Abandoning Radical Hysterectomy in Early-Stage Lymph Node-Positive Cervical Cancer: Impact on Disease-Free Survival and Treatment-Related Toxicity.[J] .Int. J. Gynecol. Cancer, 2017, 27: 1015-1020.

[15]. Garg Gunjal, Shah Jay P, Liu J Rebecca, Should radical hysterectomy be aborted on intraoperative detection of nodal tumor metastasis in early stage cervical cancer?[J] .J Low Genit Tract Dis, 2010, 14: 374-81.

[16]. Ramirez P, Frumovitz M, Pareja R, et al. Minimally invasive versus abdominal radical hysterectomy for cervical cancer囚J囚. N Engl J Med, 2018, 379(20): 1895-1904.

[17]. van de Lande Jonas, von Mensdorff-Pouilly Silvia, Lettinga Roelof $\mathrm{G}$ et al. Open versus laparoscopic pelvic lymph node dissection in early stage cervical cancer: no difference in surgical or disease outcome. [J] .Int. J. Gynecol. Cancer, 2012, 22: 107-14.

\section{Figures}

\section{Figure 1}

Kaplan-Meier analysis of progression-free survival(PFS) and overall survival(OS). 